\title{
SYSTEM IDENTIFICATION BASED INVERSE KINEMATICS ANALYSIS AND CONTROL OF A MULTI-DOF ROAD SIMULATOR
}

UDC: 531.1

Original scientific paper

https://doi.org/10.18485/aeletters.2019.4.3.4

\author{
Abdullah Çakan $^{1 *}$, Ümit Önen ${ }^{2}$ and Fatih Mehmet Botsalı ${ }^{1}$ \\ ${ }^{1}$ Konya Technical University, Faculty of Engineering and Natural Sciences, Mechanical Engineering \\ Department, Konya, Turkey \\ ${ }^{2}$ Necmettin Erbakan University, Faculty of Engineering and Architecture, Mechatronics Engineering \\ Department, Konya, Turkey
}

\begin{abstract}
:
This paper presents, system identification based inverse kinematics analysis and control of a multi-DOF road simulator. Multi-DOF road simulators are special devices which are widely used to perform road profiles to a vehicle in laboratory environments. In this paper a road simulator mechanism is virtually designed and inverse kinematics analysis is derived without using any mathematical expression. System identification method is used for inverse kinematics analysis and then controlled to perform desired positions of the vehicle wheel center point.
\end{abstract}

\section{ARTICLE HISTORY}

Received: 20.09.2019.

Accepted: 27.09.2019.

Available: 30.09 .2019

\section{KEYWORDS}

Road simulator, PID control, system identification, $c 0-$ simulation, ADAMS, MATLAB

\section{INTRODUCTION}

Road simulators are special devices which are widely used to perform road profile to a vehicle in laboratory environments. Besides the physically using these devices, it is possible to create virtual prototypes [1]. Virtual prototyping allows the researchers investigating road profile effects to vehicles by reducing costs eliminating the physical prototypes. Inverse kinematics is to obtain the joint angles for a desired trajectory or position of the end point the manipulators [2]. There are many studies in literature about deriving inverse kinematics of the manipulators and mechanisms by using different methods. Such as optimization algorithms, neural networks, neuro-fuzzy inference systems and computer aided methods [3-6]. After the inverse position analysis, controller is designed and applied to simulate the desired path for manipulators or the mechanisms $[7,8]$.

\section{MATERIAL AND METHODS}

In this paper, a multi-DOF road simulator is designed based on part of MTS Model 329 [9]. This designed system consists of three different mechanisms. These mechanisms allow to obtain horizontal, vertical and rotational motions of wheel center of the vehicle. Designed virtual prototype of the system is simulated using MSC ADAMS that is a multi-body modeling software and co-simulation is used to derive inverse kinematics and control of the system [10]. Created multi-body model of the system is shown in Fig.1.

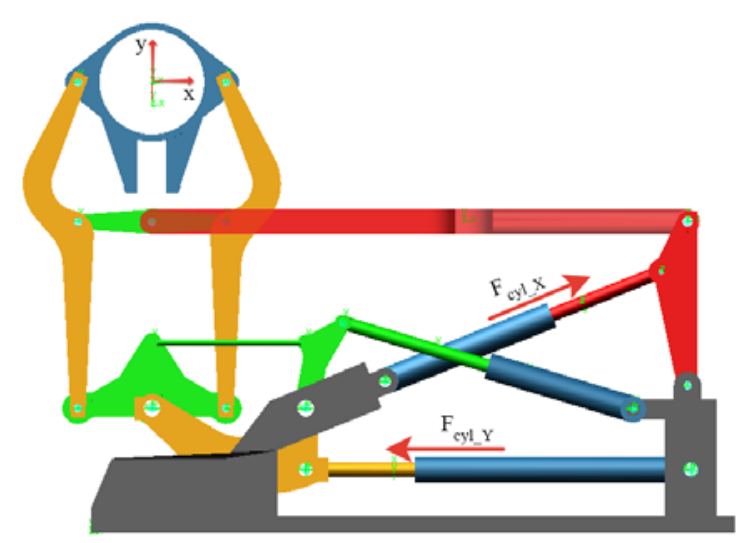

Fig.1. Multi-body model of the multi-DOF road simulator 


\subsection{System Identification based inverse kinematics}

Designed system consist of mechanisms and inverse position analysis of these mechanisms is solved as inverse kinematics problem of manipulators. In the road simulator mechanism, it is to obtain the slider displacement of inverted slider crank mechanism for desired positions of the point that is in wheel center of the vehicle. This paper presents inverse kinematics and control for only horizontal and vertical motion positions. Horizontal and vertical positions are defined as $X$ and $Y$ respectively. $X$ and $Y$ positions are obtained by applying input positions to the hydraulic cylinders. Cylinder positions are defined as the position of cylinder which creates the $X$ motion and the position of the cylinder which creates the $Y$ motion, $X_{c y l}$ and $Y_{c y l}$ respectively. It is possible to add an ADAMS plant to MATLAB/Simulink models. Therefore, these system variables are defined in the ADAMS and exported to MATLAB Software. ADAMS and MATLAB co-simulation is used to apply inputs and collecting the output data. Then, System Identification Toolbox is used to create linear inverse kinematics transfer function models of the mechanisms from input and output data [11]. Created inverse kinematics transfer models for the $X$ and $Y$ motions are shown in equations (1) and (2) respectively.

$\frac{X_{c y l}(s)}{X(s)}=\frac{-63,87313 s-0,00809}{s^{2}+159,31726 s+6,30503.10^{-6}}$

$$
\frac{Y_{c y l}(s)}{Y(s)}=\frac{-64,06041 s-62,43334}{s^{2}+159,65592 s+156,13516.10^{-6}}
$$

\subsection{Control Study}

Position control of wheel center point of the vehicle is also studied in this paper. Created inverse kinematics models are used to derive cylinder positions for the desired position of wheel center of the vehicle. PID controller is one of the most common controller types in control engineering problems, it is easy to implement and gives optimal responses by tuning proportional, integral and derivative coefficients [12]. Therefore, PID controllers are designed to achieve cylinder positions for the desired path and applied to the system. Cylinder positions are obtained by applying hydraulic forces, so cylinder forces are the output of PID controllers. $F_{\text {cyl_x }}$ and $F_{\text {cyl_y }}$ are the hydraulic forces that are applied to the cylinders to create cylinder positions. From the cylinder positions, $X$ and $Y$ positions of wheel center of the vehicle is generated using inverse kinematics models respectively. Control study is also simulated using ADAMS and MATLAB cosimulation. Block diagram of co-simulation control process is shown in Fig.2. MATLAB is sending forces the ADAMS to simulate the mechanical system and MATLAB send results to MATLAB back for the control. Results of the control are given in the results and discussion section.

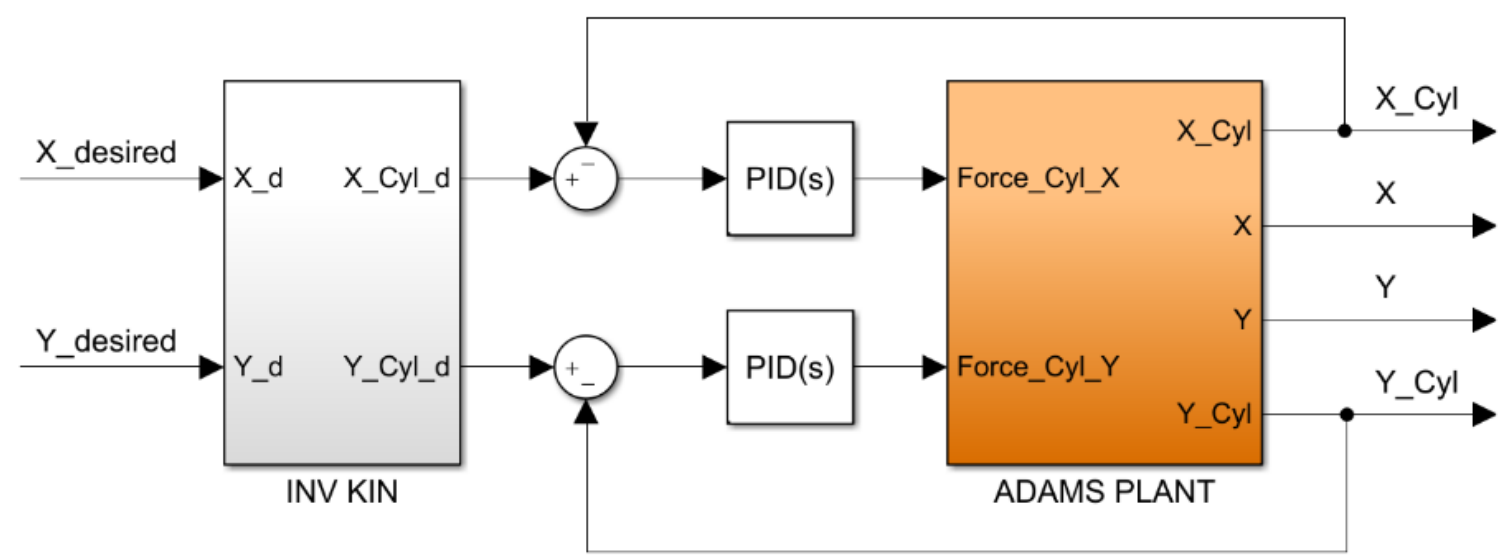

Fig.2. Closed-loop co-simulation control block diagram of the system 


\section{RESULTS AND DISCUSSION}

Inverse kinematics models of the multi-DOF road simulator are obtained using System Identification method. These models are used to derive cylinder positions and control desired path by applying hydraulic forces as output of controllers. Low frequency sinusoidal paths are desired for positions of wheel center of the vehicle. Manually tuned PID controllers achieved the desired positions with small errors. This paper presents possibility to control the proposed mechanism. Controllers can be improved for the high frequency desired paths. According to these results, it can be said that System Identification method is a useful option to create inverse kinematics models, ADAMS and MATLAB cosimulation is an efficient and accomplished way for simulating and control this kind of mechanical systems. Compared control results for the $X$ and $Y$ positions are shown in Fig.3. And Fig.4. respectively.

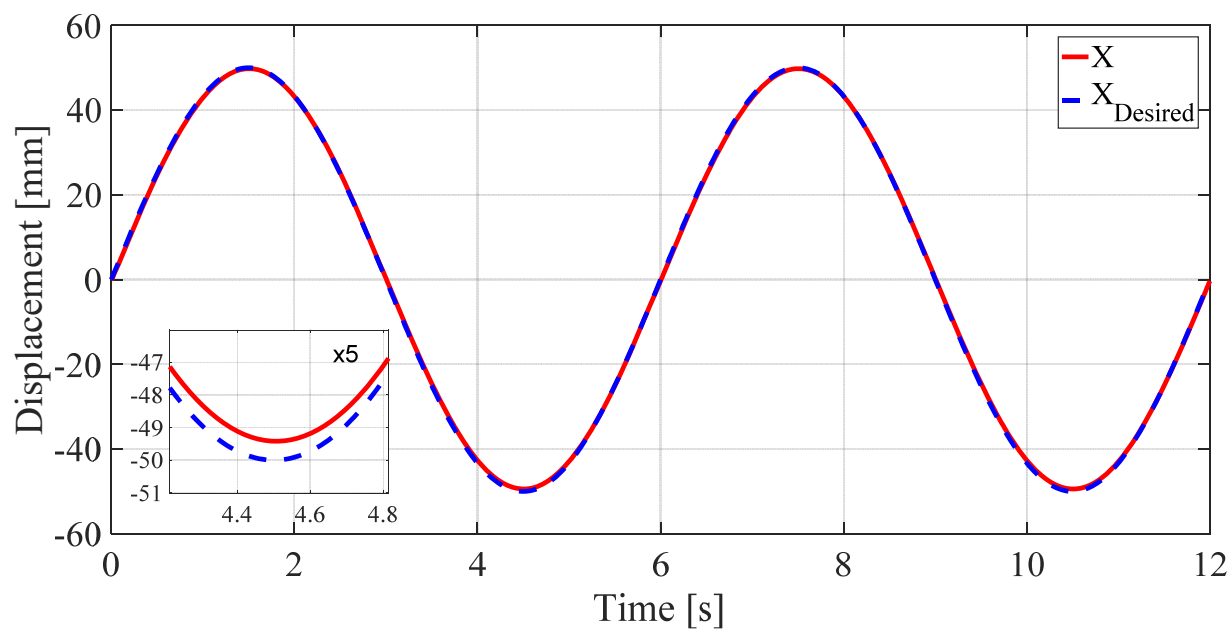

Fig.3. Compared position results for $\mathrm{X}$ motion

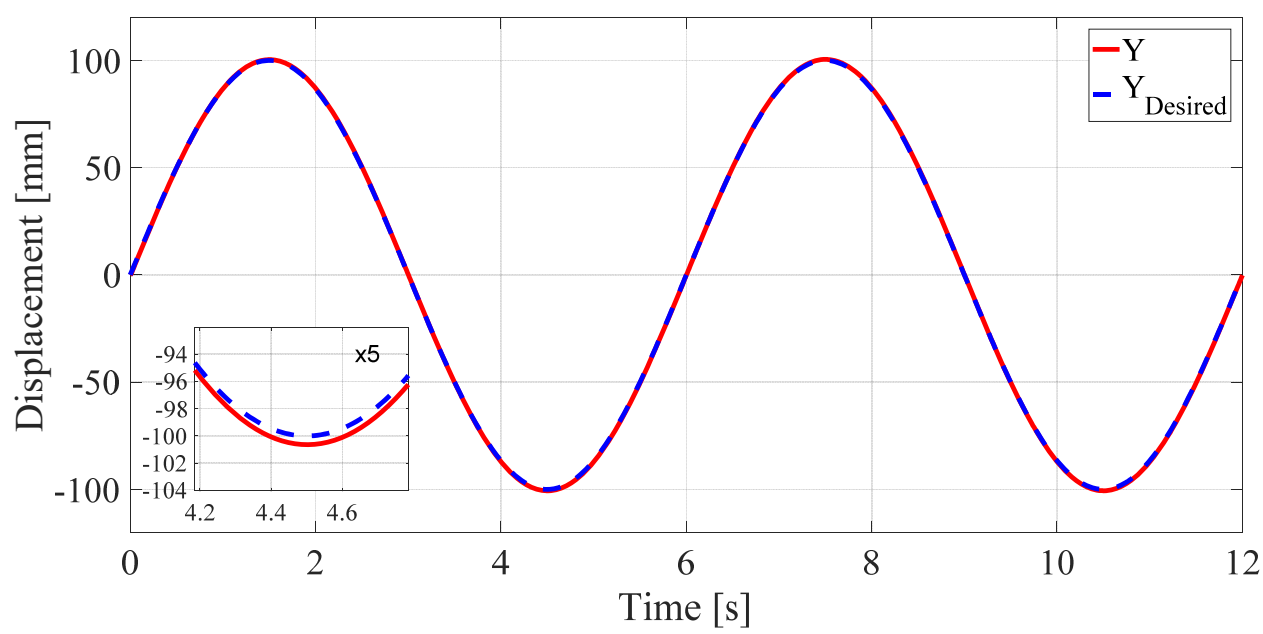

Fig.4. Compared position results for $Y$ motion

\section{CONCLUSION}

In this paper, System Identification based inverse kinematics analysis of a multi-DOF road simulator is proposed. The proposed method is another approach for inverse kinematics analysis.
Cylinder positions are derived without using any mathematical expression for the desired $X$ and $Y$ position of wheel center point of the vehicle using proposed inverse kinematics analysis. However, PID controllers are designed and applied to obtain desired $X$ and $Y$ positions. ADAMS and MATLAB co- 
simulation is used to obtain input and output data for System Identification process and implement controllers. For better responses and high frequency desired paths, optimization algorithms can be used to tune PID controller or different controllers can be designed. It is concluded that System Identification approach can be used as a method for creating inverse kinematics models and it is easy to implement controllers to this kind of mechanical systems using co-simulation.

\section{REFERENCES}

[1] K. J. Dittmann, F. J. Albrigh, C. Leser, Validation of virtual prototypes via a virtual test laboratory. Korea Institute of Materials Science $_{\mathbf{L}} 14$ (3), 2003: 27-35.

[2] Internet source: Inverse Kinematics: https://en.wikipedia.org/wiki/Inverse kinematics

[3] Alavandar, S., M. J. Nigam, Inverse kinematics solution of 3DOF planar robot using ANFIS. Int. J. of Computers, Communications \& Control, 3, 2008: 150-155.

[4] A. Çakan, F. M. Botsali, Inverse kinematics analysis of a puma robot by using MSC Adams, VI International Conference Industrial Engineering and Environmental Protection (IIZS 2016), 13-14 October, 2016, Zrenjanin, Serbia, pp.274-277.

[5] D. Pham, M. Castellani, A. Fahmy, Learning the inverse kinematics of a robot manipulator using the bees algorithm, $6^{\text {th }}$ IEEE International Conference on Industrial Informatics, IEEE, 13-16 July 2008, Daejeon, South Korea, pp.493-498: IEEE.
http://dx.doi.org/10.1109/INDIN.2008.4618151

[6] L.-X. Wei, H.-R. Wang, Y. Li, A new solution for inverse kinematics of manipulator based on neural network, Proceedings of the 2003 International Conference on Machine Learning and Cybernetics. IEEE, Vol.2, 5 November 2003, Xi'an, China, pp.1201-1203:

https://doi.org/10.1109/ICMLC.2003.1259668

[7] S. Engleder, Time-optimal motion planning and control of an electrohydraulically actuated toggle mechanism. Mechatronics, 17 (8), 2007: 448-456.

https://doi.org/10.1016/j.mechatronics.2007.05.003

[8] S. M. C. Netto, M.S. Dutra, A. Evsukoff, Fuzzy systems to solve inverse kinematics problem in robots control: application to an hexapod robots' leg, in Proceedings. Vol.1. Sixth Brazilian Symposium on Neural Networks. IEEE, 2000, pp. 150-155.

https://doi.org/10.1109/SBRN.2000.889730

[9] Internet source: MTS Model 329 Road Simulators: https://www.mts.com

[10] S. Illgen, A. Durdu, E. Gülbahçe, A. Çakan, Sliding Mode Control of a Two-link Robot Manipulator Using Adams \& Matlab Software, $6^{\text {th }}$ International Conference on Control Engineering \& Information Technology (CEIT). IEEE, 25-27 October 2018, Istanbul, Turkey, pp.1-4.

[11] L. Ljung, System identification toolbox: User's guide. The MathWorks, Inc., 1995.

[12] K. J. Åström, T. Hägglund, PID controllers: theory, design, and tuning. Instrument society of America Research Triangle Park. NC, 1995. 